\title{
Time resolved transient circular dichroism spectroscopy using synchrotron natural polarisation
}

François Auvraya,b, David Dennetiere ${ }^{\mathrm{b}}$, Alexandre Giuliani ${ }^{\mathrm{b}, \mathrm{c}}$, Frédéric Jamme ${ }^{\mathrm{b}}$, Frank Wien ${ }^{\mathrm{b}}$, Bastien Nay $^{d}$, Séverine Zirah ${ }^{\mathrm{e}}$, François Polack ${ }^{\mathrm{b}}$, Claude Menneglier ${ }^{\mathrm{b}}$, Bruno Lagarde ${ }^{\mathrm{e}}$, Jonathan D. Hirst ${ }^{\mathrm{a}}$, Matthieu Réfrégiers ${ }^{b}$ *

a School of Chemistry, University of Nottingham, University Park, Nottingham NG7 2RD, United Kingdom b Synchrotron SOLEIL, L'Orme des Merisiers, Gif-sur-Yvette 91192, France

c UAR 1008 CEPIA, INRA, Nantes 44316, France

d. Laboratoire de Synthèse Organique, CNRS UMR 7652, Ecole Polytechnique, Palaiseau 91128 Cedex, France e. Unité Molécules de Communication et Adaptation des Microorganismes (MCAM), Muséum national d'Histoire naturelle, CNRS, Paris 75005, France

f. Brain Physiology Lab - CNRS UMR 8118, Université Paris Descartes, 45 Rue des Saints Pères 75270 Paris

Cedex 06, France

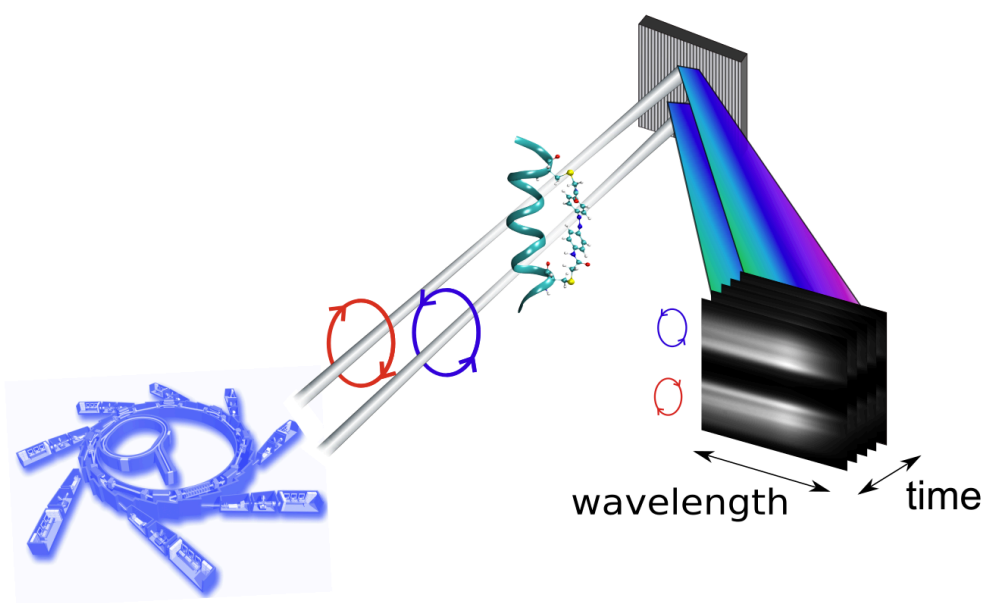

\begin{abstract}
Ultraviolet (UV) synchrotron radiation circular dichroism (SRCD) spectroscopy has made an important contribution to the determination and understanding of the structure of biomolecules. In this paper, we demonstrate an innovative time-resolved SRCD (tr-SRCD) technique, overcoming limitations of current broadband UV SRCD setups, to access ultrafast (down to nanoseconds) time-scales, previously measurable only by other techniques, such as infrared (IR), nuclear magnetic resonance (NMR), fluorescence and absorbance spectroscopies and small angle X-ray scattering (SAXS). The tr-SRCD setup takes advantage of the natural polarisation of the synchrotron radiation emitted by a bending magnet to record broadband UV CD faster than any current SRCD setup, improving the acquisition speed from $10 \mathrm{mHz}$ to $130 \mathrm{~Hz}$ and the accessible temporal resolution by 11 orders of magnitude. We illustrate the new approach by following the photoisomerization of an azopeptide. This breakthrough in SRCD spectroscopy opens up a wide range of potential applications to the detailed characterisation of biological processes, such as protein folding, protein-ligand binding and DNA nano-structure formation.
\end{abstract}

Keywords: time-resolved spectroscopy, circular dichroism, synchrotron, biomolecular dynamics

\footnotetext{
○Corresponding author: matthieu.refregiers@synchrotron-soleil.fr
} 


\section{Introduction}

Circular dichroism (CD) is an optical property of molecules having chiral structure(s) and/or spatially oriented arrays of chromophores. It manifests as a difference in absorption for left- and right-circularly polarised light. In the ultraviolet (UV) range, this feature has been exploited for many decades for characterisation of organic molecules, materials with supramolecular chirality, and in protein conformation determination, where there are distinctive spectral signatures for each secondary structure type, i.e., $\alpha$-helices and $\beta$-sheets ${ }^{1}$. Thus, CD spectroscopy is an important biophysical tool for characterising native and modified proteins. In the biomedical context, protein misfolding can have dramatic consequences on cell physiology, causing serious neurodegenerative diseases, as found in Alzheimer's and Parkinson's ${ }^{2}$. Structural and kinetics studies of protein folding, using time-resolved approaches, are providing crucial insights at the molecular level into the aetiology of these diseases.

There are two main ways to measure CD spectra: the ellipsometric method and the direct absorption difference detection method. The former is based on quantifying the variations of the ellipticity and azimuth orientation of a highly elliptically polarised beam passing through a dichroic sample ${ }^{3}$. In 2012, Eom et al. ${ }^{4}$ innovatively adapted the ellipsometric method to a heterodyne-detection technique, providing both the CD spectrum and the optical rotation dispersion spectrum, by analysis of the phase and the amplitude of the transmitted orthogonal electric field of the incident light polarisation. More recently, Hiramatsu et al. ${ }^{5}$ coupled the heterodyne detection technique to a singular value decomposition analysis. This improvement removes linear dichroism and linear birefringence artifacts, allowing accurate time-resolved CD (tr-CD) measurements in the visible range with sub-picosecond temporal resolution. The second way to acquire a CD spectrum is based on absorption difference 
measurement. The light is alternately circularly right and left polarised using an optical or acousto-optic modulator. Then the detection system records intensity variations and allows CD determination. This method has been extensively refined, and can provide sub-picosecond resolution for monochromatic measurements. Hache and colleagues used this technique to probe ultra-fast kinetics in bio-molecules ${ }^{6}$ and achieved 800 fs temporal resolution ${ }^{7}$. Trifonov et al. ${ }^{8}$ have developed a setup for picosecond transient broadband CD measurements in the visible range. They used a combination of a Pockels cell and a light continuum generator to obtain a pulsed white light source alternately circularly right and left polarised. Both ellipsometric and absorption difference broadband techniques have advantages and disadvantages, but they also share the same spectral restriction to the visible range, due to the lack of stable continuum light sources in the UV range. This limits the application of these broadband tr-CD spectroscopies and makes them unsuitable for UV CD measurements.

The synchrotron radiation circular dichroism (SRCD) technique was developed in 1980 9,10 . Since its first use for protein structure determination ${ }^{11}$, it has been used in a wide range of applications ${ }^{12-14}$. Indeed, the brilliance of the synchrotron radiation (SR) in the vacuum UV (VUV) range and its stability enables one to measure CD spectra of samples dissolved in buffer down to $160 \mathrm{~nm}^{15}$ with an acceptable signal to noise ratio for the heterodyne detection.

In this paper, we describe the development of a different approach for SRCD measurements, utilising the natural polarisation of the SR emitted by a bending magnet ${ }^{16,17}$. The DISCO beamline provides a SR beam composed of two parts (Supplementary Information Fig. 1), which are above and below the accelerated electron's orbital plane in the synchrotron storage ring. These two continuum beams are similarly elliptically polarised but with opposite direction (Supplementary Information 2). Schiller and Hormes ${ }^{18}$ have previously demonstrated that the natural elliptical polarisation of the SR can be used for CD spectroscopy. However, they measured the CD signal sequentially, wavelength by wavelength; so the scanning process 
of the monochromator limited the temporal resolution of their setup significantly. We have developed a spectrograph that measures simultaneously the intensity of both (oppositely polarised) parts of the SR beam, allowing one to determine a whole UV CD spectrum in just a single measurement. While current SRCD setups only take advantage of the wide spectral range and the brilliance of SR, our setup also uses its natural polarisation and its temporal distribution. This broadband single measurement approach gives access to a combination of a temporal and a spectral range previously inaccessible with the current broadband CD setups, enabling new insights into biomolecular dynamics.

\section{Method}

The tr-SRCD setup is annexed to the existing SRCD endstation at the DISCO beamline 19. The optical layout is shown Figure 1. The beamline excitation monochromator is used first order for the wavelength calibration of the detection spectrograph (Supplementary Information Fig. 3). With the zero order, we get on the sample a pulsed white beam containing all the wavelengths between $120 \mathrm{~nm}$ to $600 \mathrm{~nm}$; this pulsed broadband source is used for the tr-SRCD measurement, limited by the CMOS spectral response. The beam is spatially defined by a spatial filter (labelled S1 in the figure), then centred by the two plane mirrors (M1, M2) before being refocused on the sample. In order to avoid damage from beam irradiation, this optical system is designed to allow variation of the illuminated area on the sample. The spherical mirror M3 and the cylindrical mirror M4 refocused the beam $2975 \mathrm{~mm}$ after the monochromator slits; the calculated beam diameter at the focal plane is about $300 \mu \mathrm{m}$ (FWHM). By moving the sample cell by $200 \mathrm{~mm}$ along the optical axis, we get a controllable probe beam diameter from $300 \mu \mathrm{m}$ up to $3 \mathrm{~mm}$. Adjustment of the beam diameter to the exposure time helps to minimize the sample irradiation dose. Beyond the sample, the cylindrical mirror M5 and the spherical mirror M6 horizontally focused the beam on a 
secondary slit (S2). This secondary slit at $3900 \mathrm{~mm}$ is used to define the final spectral resolution of the setup. The flat M7 mirror reflected the beam on a spherical flat field grating with 580 grooves per mm (Horiba Jobin-Yvon) that diffract and focus the incident UV/vis light horizontally onto the $2 \mathrm{D}$ detector.

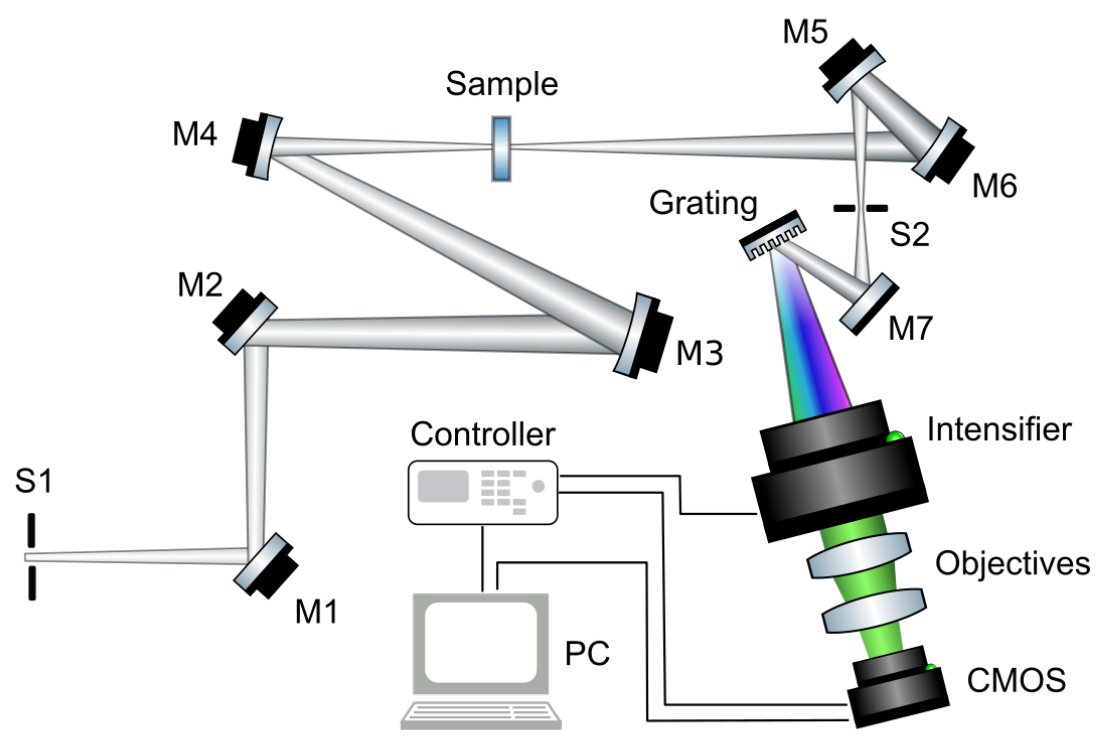

Figure 1: Optical layout of the tr-SRCD setup.

The tr-SRCD setup acquisition frequency is defined by the camera frame rate, which can be set from $0.033 \mathrm{~Hz}$ to $100 \mathrm{~Hz}$ in full resolution mode and can be increased further by reducing the pixel array size. This range of sampling frequency allows one to study reaction kinetics from tens of milliseconds to minutes through real-time recording and sequential measurements. The image intensifier can be synchronized with the camera output clock and with an external function generator providing trigger signals with a higher frequency. When the intensifier is synchronized with the output clock, the temporal resolution of the measurement 
corresponds to the duration of the amplification gate or the light pulse length if the gate is sufficiently short (Supplementary Information 4). However, if an external device triggers the image intensifier at a higher frequency than the camera frame rate, the exposure time of the camera defines the temporal resolution of the setup.

The sample was held in a $\mathrm{CaF}_{2}$ flow cell with a path-length of $24 \mu \mathrm{m}$ (Aquaspec, Microbiolytics GmbH, Freiburg, D). The sensitivity ranges of 2D detectors, such as CDD or CMOS, are usually limited to $200 \mathrm{~nm}$. We may also use an image intensifier (HS-IRO, LAVISION) to reach lower wavelength range and to increase time resolution. To recover the main part of the visible light provided by the HS-IRO phosphor, a combination of two $50 \mathrm{~mm}$ focal length infinity corrected objectives was used. A CMOS detector (Prime 95-B, PHOTOMETRICS) records the phosphor screen image. For the applications that do not need the picosecond temporal resolution, the Prime 95-B was used without intensifier.

Equation 1 defines $\mathrm{CD}$ at a given wavelength. The variables $A_{L}$ and $A_{R}$ correspond to the absorption for left and right-handed circularly polarised light, respectively. All the spectra associated with the tr-SRCD setup in this study were determined by the following equation.

$$
C D=\left(A_{L}-A_{R}\right) \quad \text { Equation } 1
$$

In this case, the absorption difference between left-handed and right-handed circularly polarised light can be associated with the absorption difference between the upper and lower part of the SR (Supplementary Information Note 5).

\section{Results}

We chose camphorsulfonic acid (CSA), purchased from Sigma-Aldrich, as our calibration sample, as it has two strong peaks in the VUV/deep UV range: the first at $290 \mathrm{~nm}$ and the second at $192 \mathrm{~nm}$. It is commonly used as a calibration standard for CD spectrometers 
${ }^{20,21}$. To validate that the recorded signal is $\mathrm{CD}$, the two enantiomers must exhibit opposite signed spectra.

The resulting spectra for D-CSA and L-CSA, measured between $190 \mathrm{~nm}$ and $315 \mathrm{~nm}$ for $30 \mathrm{mg} / \mathrm{mL}$ solution concentration, are shown in Figure 2A. The CMOS detector has been used without the image intensifier. It integrated the light for $500 \mu$ s and the images were acquired at $20 \mathrm{~Hz}$. As expected, we observe two oppositely signed spectra with identical amplitudes. The ratio between the amplitude of the two peaks should be about $2^{21}$; we obtained $2.11+/-0.11$ in the tr-SRCD spectra (curve (d) of Figure 2A). The signal-to-noise ratios at $190 \mathrm{~nm}$ for the DCSA spectra (a), (b), (c) and (d) in Figure 2A are 6.6, 12.7, 39.8 and 44.40, respectively.
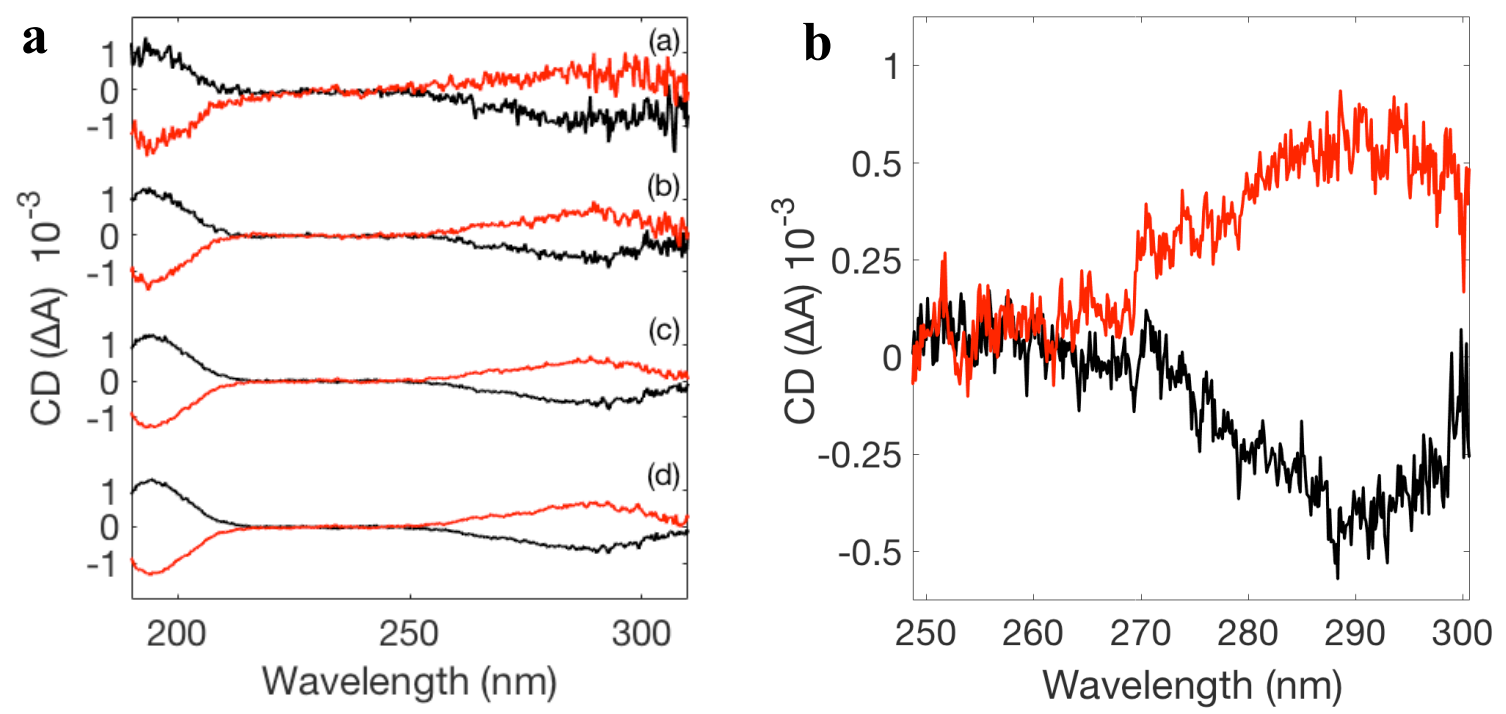

Figure 2: a. CD spectra of D-CSA (black) and L-CSA (red) acquired with the tr-SRCD setup at $20 \mathrm{~Hz}$ with $500 \mu$ s temporal resolution. 1, 10, 50 and 100 measurements were integrated to obtain the data shown in curves (a), (b), (c) and (d) respectively. b. CD spectra of D-CSA (black) and L-CSA (red) from single pulse measurements acquired with the tr-SRCD setup at $500 \mathrm{~Hz}$ with 82 ps temporal resolution; 5000 measurements were integrated.

In order to establish that the highest theoretical temporal resolution of the setup is attainable experimentally, we added an image intensifier, thereby obviating the temporal 
resolution limitation of the CMOS detector. We performed this steady state experiment on the CSA with the maximal temporal resolution as a proof-of-principle. The intensifier was triggered with an external trigger and its amplification was reduced to its shortest value, $100 \mathrm{~ns}$. The measurements were made using the 8 bunch mode of the synchrotron SOLEIL which provides 82 ps pulses at $7.14 \mathrm{MHz}$. The intensifier amplified only one pulse per gate. So its temporal resolution corresponds to the pulse length, which, in this case, is 82 ps. In Figure 2B, each spectrum comes from ten images; 500 separated pulses were integrated for each image. The spectral range was reduced to between $255 \mathrm{~nm}$ and $300 \mathrm{~nm}$, in order to optimize the setup for the detection of the peak at $290 \mathrm{~nm}$. The signal-to-noise ratio at $290 \mathrm{~nm}$ for the D-CSA spectrum is 10.33 .

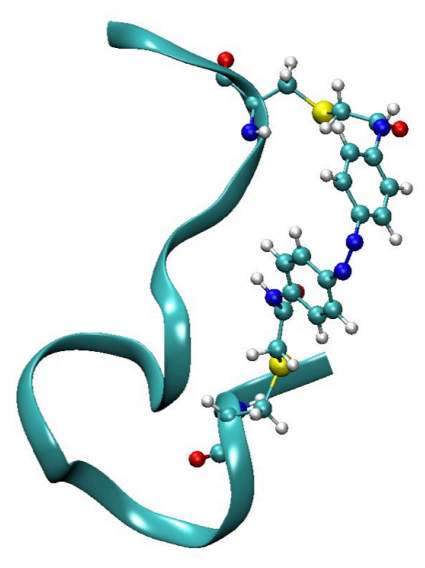

$460 \mathrm{~nm}$

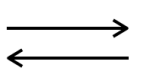

$370 \mathrm{~nm}$

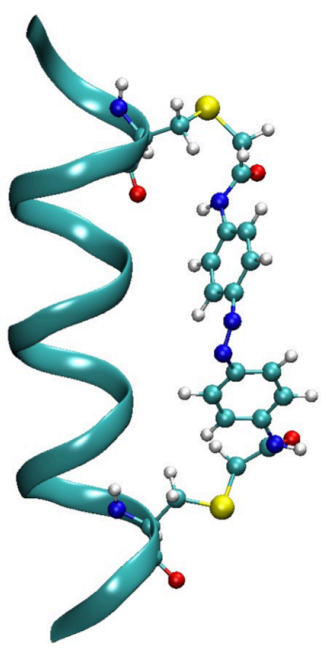

Figure 3: Schematic representation of the FK-11-X molecular system for both cis and trans conformation. Its amino acid sequence is Ac-Glu-Ala-Cys ${ }^{\mathrm{AZO}}$-Ala-Arg-Glu-Ala-Ala-Ala-Arg-Glu-AlaAla-Cys ${ }^{\mathrm{AZO}}$-Arg-Gln- $\mathrm{NH}_{2}$.

We tested the capacity of the setup to follow reactions in real time by studying a photocontrolled and reversible system, the azobenzene crosslinked peptide FK-11- $\mathrm{X}^{22,23}$. It is 
composed of a 16 amino acid peptide cross-linked to a photoswitchable molecule, the azobenzene (Figure 3). This ligand can be isomerised from the trans to cis and cis to trans conformation using a $370 \mathrm{~nm}$ and a visible light source $(460 \mathrm{~nm})$, respectively. Several groups have studied the dynamics of this photo-induced reaction and the isomerisation of the azobenzene appears to occur within one picosecond ${ }^{24-27}$. This conformational change constrains the peptide structure and triggers its folding and unfolding processes. Theoretical 22,28 and experimental ${ }^{29,30}$ studies agree that, following the azobenzene isomerisation, peptide conformational changes occur on the microsecond scale. The azobenzene cross-linked peptide was synthesized as described previously ${ }^{22}$ (Supplementary Information Note 6). The aim of our study was to follow the change in concentration of the unfolded (cis azobenzene) and folded (trans azobenzene, ground-state) FK-11-X peptide. We triggered the isomerisation with two continuous diodes, at $370 \mathrm{~nm}(5 \mathrm{~W})$ and at $460 \mathrm{~nm}(5 \mathrm{~W})$. The concentration of FK-11-X solution was $10 \mathrm{mg} / \mathrm{mL}$ in phosphate buffer $(70 \mathrm{mM}, \mathrm{pH} 7)$. The sample was alternately irradiated with the two diodes, for 2.5 seconds for each. The transient CD and absorption spectra of the cis to trans isomerisation were followed, measuring spectra at $130 \mathrm{~Hz}$ with a 500 $\mu$ s temporal resolution; the measurement has been integrated over 20 cycles. The variations in the $\mathrm{CD}$ and absorption are shown in Figure 4. 

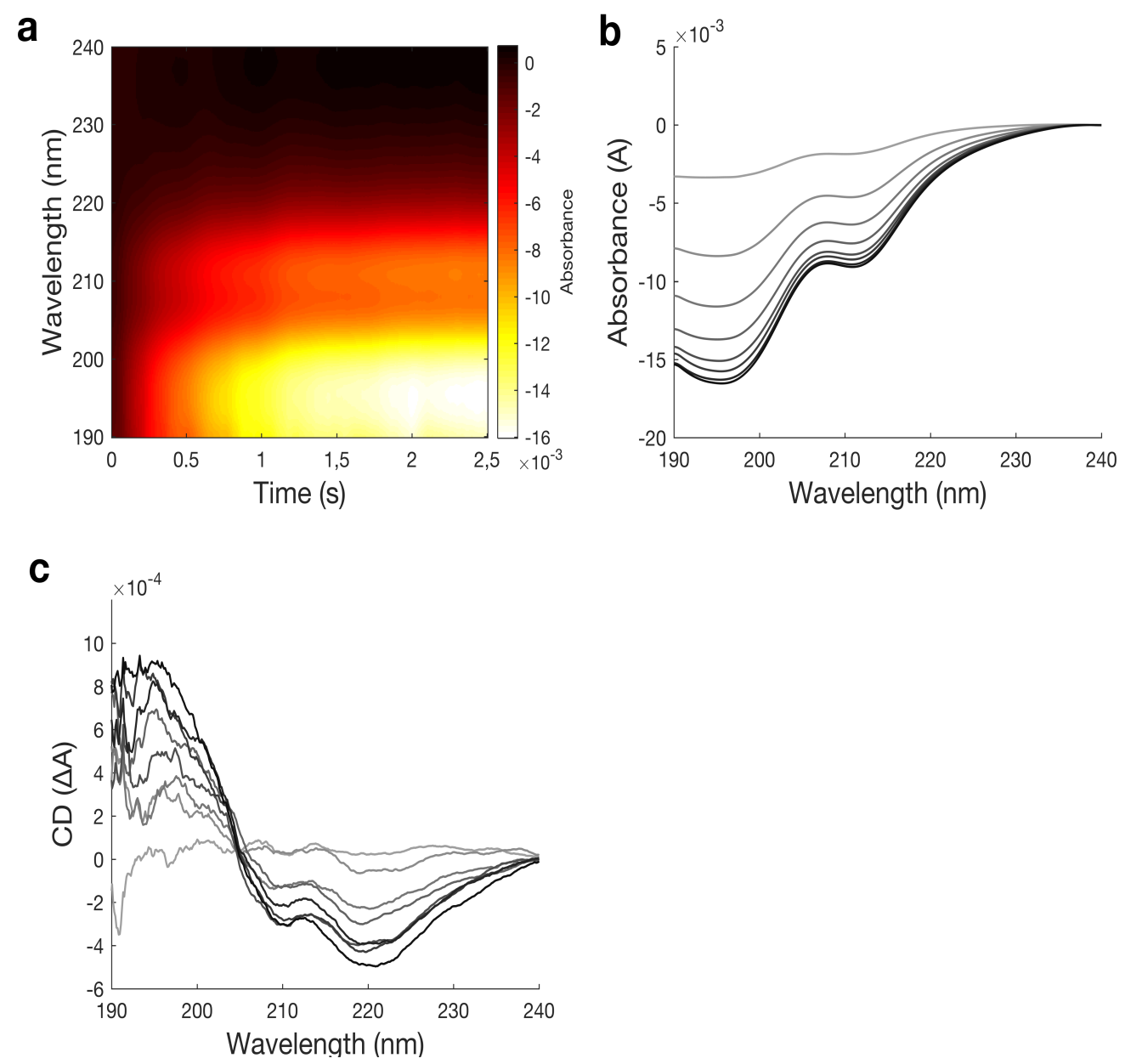

Figure 4: a. 2D representation of the evolution of the absorbance over time; 650 spectra are shown. $\mathbf{b}$. Concatenation of the 650 absorption spectra into 8 averaged spectra. c. Evolution of the CD over time with a $425 \mathrm{~ms}$ time-step.

We observe a decrease in the concentration of the cis-conformation species from the first milliseconds, as shown by the CD and the absorption changes (Figure 4). The absorption decreases progressively and reaches a plateau after the 2.5 seconds of irradiation with the 460 $\mathrm{nm}$ diode. The $\mathrm{CD}$ spectrum corresponds to the signal of the $\alpha$-helical structure and the increasing amplitude reflects the change of the species concentration, from cis to transconformation, i.e., from unfolded to folded structures. The total CD changes from the initial state and final state measured with the tr-SRCD setup and from steady state measurement (cis and trans $\mathrm{CD}$ spectra) in the literature ${ }^{29}$ are similar. This comparison shows that being able to 
calibrate the SR polarisation, we can measure transient CD spectra similar to those measured with steady state setups. The only difference arising from the elliptical polarisation is the amplitude of the $\mathrm{CD}$, which is linearly correlated to the circularity of the polarisation.

On going experiments involve the kinetic study of the refolding of model proteins after thermal denaturation.

Our study demonstrates that the new tr-SRCD setup developed at the DISCO beamline 19 in the SOLEIL synchrotron offers new opportunities for the investigation of biomolecular structure and dynamics. The setup is based on the use of the natural properties of the synchrotron radiation provided by the DISCO beamline. It uses both spectral and polarisation characteristics to reduce the time required for the measurement of a transient CD UV spectrum. Our tr-SRCD measurements of the CSA show that an integration time of $500 \mu$ s is enough to obtain an acceptable signal-to-noise ratio, i.e., greater than 6 . We performed a proof-ofprinciple high temporal resolution steady state experiment on CSA performed using an image intensifier allowing resolution down to a few tens of picoseconds. The FK11 experiment demonstrates the capacity of the tr-SRCD setup to follow dynamics in real time, with a temporal resolution of $500 \mu \mathrm{s}$ and acquired at $130 \mathrm{~Hz}$. These values do not correspond to the hardware cap and can be easily improved, by opening the spectrograph slits and integrating more photons in shorter time period and/or coupling the system with the image intensifier. This breakthrough in the SRCD spectroscopy will enable the study of many biological and chemical reactions crucial for our understanding of biomolecular phenomena.

The development of new CaF2 microlenses windows for sCMOS camera would grant access to direct detection and measurement of spectra on the full DUV range down to $160 \mathrm{~nm}$.

In the near future, the setup could easily be coupled to other reaction triggers, such as a T-jump, lasers and microfluidic stop-flow equipment. Thus, this new approach will provide 
exciting insights into the dynamics of biomolecules, as well as for molecular and materials systems more broadly. This method will enable one to follow the behaviour of molecules through high quality SRCD spectra on a temporal range from the picoseconds to minutes. Indeed, it covers time scales consistent with the fluctuation and domain motions of proteins suspected to play a fundamental role in their activity.

Acknowledgment Access to synchrotron SOLEIL was supported under project \#20151217, \#20161257, \#20161315. We thank the University of Nottingham and SOLEIL for a PhD studentship for F.A.

Author Contributions M.R., F.W., F.J. and A.G. came with the original idea. M.R., A.G. and J.D.H. conceived the research. F.P., B.L., D.D., and F.A. conceived the optical scheme. F.A., A.G., F.W. and F.J. performed the experiments. B.N. and S.Z. conceived and prepared the peptides samples. C.M. conceived the detection scheme. F.A., J.D.H., A.G. and M.R. wrote the manuscript. All authors commented on the drafts of the manuscript.

\section{Competing Interest None.}

Data availability statement Raw data were generated at the synchrotron SOLEIL large scale facility and are available from the corresponding author upon reasonable request.

\section{References}

1. Greenfield, N. \& Fasman, G. D. Computed circular dichroism spectra for the evaluation of protein conformation. Biochemistry 8, 4108-4116 (1969).

2. Hartl, F. U. Protein misfolding diseases. Annu. Rev. Biochem. 86, 21-26 (2017).

3. Zhang, C. F., Lewis, J. W., Cerpa, R., Kuntz, I. D. \& Kliger, D. S. Nanosecond circular dichroism spectral measurements: extension to the far-ultraviolet region. J. Phys. Chem. 97, 5499-5505 (1993).

4. Eom, I., Ahn S. H., Rhee, H. \& Cho, M. Single-shot electronic optical activity interferometry: power and phase fluctuation-free measurement. Phys. Rev. Lett. 108, 103901 (2012).

5. Hiramatsu, K. \& Nagata, T. Broadband and ultrasensitive femtosecond time-resolved circular dichroism spectroscopy. J. Chem. Phys. 143, 121102 (2015). 
6. Dartigalongue, T. \& Hache, F. Observation of sub-100 ps conformational changes in photolyzed carbonmonoxy-myoglobin probed by time-resolved circular dichroism. Chem. Phys. Lett. 415, 313-316 (2005).

7. Dartigalongue, T., Niezboral, C. \& Hache, F. Subpicosecond UV Spectroscopy of carbonmonoxy-myoglobin: absorption and circular dichroism studies. Phys. Chem. Chem. Phys, 9, 1-5 (2007).

8. Trifonov, A., Buchvarov, I., Lohr, A., Würthner, F. \& Fiebig, T. Broadband femtosecond circular dichroism spectrometer with white-light polarization control. Rev. Sci. Instrum, 81, 043104 (2010).

9. Snyder, P. A. \& Rowe, E. M. The first use of synchrotron radiation for vacuum ultraviolet circular dichroism measurements. Nuclear Instruments and Methods 172, 345-349 (1980).

10. Sutherland, J. C., Desmond, E. J. \& Takacs, P. Z. Versatile spectrometer for experiments using synchrotron radiation at wavelengths greater than $100 \mathrm{~nm}$. Nuclear Instruments and Methods 172, 195-199 (1980).

11. Sutherland, J. C., Emrick A., France, L. L., Monteleone, D. C. \& Trunk, J. Circular dichroism user facility at the national synchrotron light source: estimation of protein secondary structure. Biotechniques 13, 588-590 (1992).

12. Miles, A. J. \& Wallace, B. A. Synchrotron radiation circular dichroism spectroscopy of proteins and applications in structural and functional genomics. Chem. Soc. Rev. 35, 39-51 (2006).

13. Bulheller, B.M., Miles, A.J., Wallace, B.A. \& Hirst, J.D. Charge-transfer transitions in the vacuum-ultraviolet of protein circular dichroism spectra. J. Phys. Chem. B. 112, 1866-1874 (2008).

14. Matsuo K., Yonehara R. \& Gekko K. Secondary-structure analysis of proteins by vacuum-ultraviolet circular dichroism spectroscopy. J. Biochem. 135, 405-411 (2004).

15. Wallace B. A. Conformational changes by synchrotron radiation circular dichroism spectroscopy. Nat. Struct. Biol. 7, 708-709 (2000).

16. Heinzmann, U., Osterheld, B. \& Schafers, F. Measurements and calculations of the circular polarization and of the absolute intensity of synchrotron radiation in the wavelength range from 40 to $100 \mathrm{~nm}$. Nuclear Instruments and Methods in Physics Research 195, 395-398 (1982).

17. Kang, B. S., Fischer, P., Kim, D. H., Attwood, D., Anderson, E. \& Cho, G. Bending magnet $\mathrm{X}$-ray polarization modulation for magnetic full-field soft $\mathrm{x}$-ray transmission microscopy. IPAP conference series 7, 288-290. (2006).

18. Schiller, J. \& Hormes, J. Using the circularly polarized components of synchrotron radiation for circular dichroism measurements between 400 and 3500A. Nucl. Instr. Meth. Phys. Res. A246, 772-775 (1986).

19. Giuliani, A., Jamme, F., Rouam, V., Wien, F., Giorgetta, J. L., Lagarde, B., Chubar, O., Bac, S., Yao, I., Rey, S., Herbeaux, C., Marlats, J. L., Zerbib, D., Polack, F. \& Refregiers, M. DISCO: a low-energy multipurpose beamline at synchrotron SOLEIL. $J$. Sync. Rad. 16, 835-841 (2009).

20. Refregiers, M., Wien, F., Ta, H. P., Premvardhan, L., Bac, S., Jamme, F., Rouam, V., Lagarde, B., Polack, F., Giorgetta, J. L., Ricaud, J. P., Bordessoule, M. \& Giuliani, A. Synchrotron radiation circular dichroism endstation at SOLEIL. J. Sync. Rad. 19, 831835 (2012).

21. Miles, A. J., Wien, F. \& Wallace, B. A. Redetermination of the extinction coefficient of camphor-10-sulfonic acid, a calibration standard for circular dichroism spectroscopy. Anal. Biochem. 335, 338-339 (2004).

22. Flint D. G., Kumita J. R., Smart O. S. \& Woolley G. A. Using an azobenzene cross- 
linker to either increase or decrease peptide helix content upon trans-to-cis photoisomerization. Chem Biol 9, 391-397 (2002).

23. Kumita J. R., Smart O. S. \& Woolley G. A. Photo-control of helix content in a short peptide. Proc. Natl. Acad. Sci. USA 97, 3803-3808 (2000).

24. Nenov, A., Borrego-Varillas, R., Oriana, A., Ganzer, L., Segatta, F., Conti, I., SegarraMarti, J., Omachi, J., Dapor, M., Taioli, S., Manzoni, C., Mukamel, S., Cerullo G. \& Garavelli M. UV-Light-Induced Vibrational Coherences: The key to understand Kasha rule violation in trans-azobenzene.. J. Phys. Chem. Lett. 9, 1534-1541 (2018).

25. Nägele, T., Hoche, R., Zinth, W. \& Wachtveitl, J. Femtosecond photoisomerization of cis-azobenzene. Chem. Phys. Lett. 272, 489-495 (1997).

26. Fujino, T. \& Tahara, T. Picosecond time-resolved Raman study of trans-azobenzene. $J$. Phys. Chem. A 104, 4203-4210 (2000).

27. Fujino, T., Arzhantsev, S. Y. \& Tahara, T. Femtosecond time-resolved fluorescence study of photoisomerization of trans-azobenzene. J. Phys. Chem. A 105, 8123-8129 (2001).

28. Xia, S. H., Cui, G., Fang, W. H. \& Thiel, W. How photoisomerization drives peptide folding and unfolding: insights from $\mathrm{QM} / \mathrm{MM}$ and $\mathrm{MM}$ dynamics simulations. Angew. Chem. Int. Ed. 55, 2067-2072 (2016).

29. Woolley, G. A. Photocontrolling peptide $\alpha$ helices. Acc. Chem. Res. 38, 486-493 (2005).

30. Chen, E., Kumita, J. R., Woolley, G. A. \& Kliger, D. S. The kinetics of helix unfolding of an azobenzene cross-linked peptide probed by nanosecond time-resolved optical rotatory dispersion. J. Am. Chem. Soc. 125, 12443-12449 (2003). 